\title{
Immune regulation of neuronal injury and repair by observing $T$ cell activation
}

Eric J Regele ${ }^{1}$, Elizabeth M Runge ${ }^{1,2}$, Felicia M Kennedy ${ }^{1,2}$, Virginia M Sanders ${ }^{3}$, Kathryn J Jones ${ }^{1,2}$

${ }^{1}$ Indiana University School of Medicine, Department of Anatomy, Indianapolis, IN, USA

${ }^{2}$ Research and Development, Richard L. Roudebush VA, Indianapolis, IN, USA ${ }^{3}$ Cell Biology and Genetics, The Ohio State University, Columbus, OH, USA

\section{Background and Hypothesis:}

It is unknown how the immune system maintains the majority of facial

motoneuron (FMN) survival after axotomy. IL-10 cytokine is necessary for FMN survival and $\mathrm{CD}^{+}{ }^{+} \mathrm{T}$ cells are activated and play a critical role in survival, but do not produce IL-10. It was proposed that the source of IL-10 resides in the CNS; however, it is possible that antigen presenting cells (APC) produce IL-10 which activate $\mathrm{CD} 4^{+} \mathrm{T}$ cells to a neuroprotective phenotype. The regulation of IL-10 receptors (IL-10R) in immunodeficient compared to wild-type (WT) mice in the facial nucleus was studied in this experiment, as well as the possibility of the PNS producing IL-10.

\section{Experimental Design or Project Methods:}

To study APC's role in motoneuron survival, we transferred WT whole splenocytes into global IL-10 knock out (KO) mice prior to axotomy. To study IL10R gene expression, immunodeficient RAG-2 KO mice received WT or IL-10R ${ }^{-1-}$ $\mathrm{CD} 4^{+} \mathrm{T}$ cells prior to axotomy.

\section{Results:}

qPCR revealed that WT mice upregulate IL-10R after axotomy, whereas RAG-2 $\mathrm{KO}$ mice had decreased expression comparatively. RAG-2 mice who received WT CD4 ${ }^{+} \mathrm{T}$ cells transfer restored IL-10R comparable to WT values. IL-10R was rescued in RAG-2 mice after the adoptive transfer of WT CD4 ${ }^{+} T$ cells. When IL$10 \mathrm{R}^{-/-}$CD $4^{+}$cells were transferred into RAG-2 mice, IL-10R values were restored; however, these T cells were unable to rescue FMN survival.

\section{Conclusion and Potential Impact:}

If WT whole splenocytes transferred into global IL-10 KO mice rescue FMN survival, it implies that APC play a role in producing IL-10. If they cannot mediate rescue, then peripheral IL-10 is unlikely sufficient for FMN survival. CD4 ${ }^{+} \mathrm{T}$ cells regulate central IL-10R response and must respond to IL-10 to mediate FMN survival. The transfer of whole splenocytes provides APCs capable of producing $\mathrm{IL}-10$ and $\mathrm{CD} 4^{+} \mathrm{T}$ cells capable of responding to IL-10. 